\title{
Erratum to: First-in-human evaluation of a hybrid modality that allows combined radio- and (near-infrared) fluorescence tracing during surgery
}

Nynke S. van den Berg ${ }^{1,2}$ • Hervé Simon ${ }^{3}$ - Gijs H. Kleinjan ${ }^{1,4}$ • Thijs Engelen ${ }^{1,5}$. Anton Bunschoten ${ }^{1}$ Mick M. Welling ${ }^{1} \cdot$ Bernard M. Tijink $^{5} \cdot$ Simon Horenblas $^{2}$ • Jacques Chambron $^{4} \cdot$ Fijs W. B. van Leeuwen ${ }^{1,2,5}$

Published online: 11 July 2015

(C) Springer-Verlag Berlin Heidelberg 2015

Erratum to: European Journal of Nuclear Medicine and Molecular Imaging

DOI 10.1007/s00259-015-3109-3

The original version of the above article contained a mistake in one of the author names. Instead of Mick N. Welling, it should be Mick M. Welling.

The online version of the original article can be found at http://dx.doi.org/ 10.1007/s00259-015-3109-3.

Fijs W. B. van Leeuwen

F.W.B.van_Leeuwen@lumc.nl

1 Interventional Molecular Imaging Laboratory, Department of Radiology, Leiden University Medical Center, Albinusdreef 2, C2-S zone, PO Box 9600, 2300 RC Leiden, The Netherlands

2 Department of Urology, The Netherlands Cancer Institute - Antoni van Leeuwenhoek Hospital, Plesmanlaan 121, 1066

CX Amsterdam, The Netherlands

3 Eurorad S.A., 2 Rue Ettore Bugatti, 67201 Eckbolsheim, France

4 Department of NuclearMedicine, The Netherlands Cancer InstituteAntoni van Leeuwenhoek Hospital, Plesmanlaan 121, 1066 CX Amsterdam, The Netherlands

5 Department of Head and Neck Surgery and Oncology,

The Netherlands Cancer Institute - Antoni van Leeuwenhoek Hospital, Plesmanlaan 121, 1066 CX Amsterdam, The Netherlands 